\title{
Nigella Sativa Immunomodulatory Activity as a Potential Treatment of Novel Coronavirus (2019-nCoV) - A Review of Current Literature
}

\author{
Issa Rasheed Fetian ${ }^{1}$, Issa D Fitian², Lukas Villiger ${ }^{3}$, Maged Darwish $^{4}$ \\ 1 University of Basel, Basel, Switzerland; HAP/MZB \& Diamon Institute, Brugg, Switzerland, ${ }^{2}$ Cyberknife-Radiosurgery Radiation Oncology Department, Hima \\ San Pablo Hospital, Caguas, Puerto Rico, ${ }^{3}$ Division of Endocrinology, Diabetes and Metabolism, Diamon Institute; Practice Dr Villiger, ${ }^{4}$ Alnylam Pharmaceutical \\ Inc., Massachusetts, USA \\ Keywords: Nigella sativa, thymoquinone, magnetic hyperthermia, endothelin-1, covid-19, sars-cov2 \\ https://doi.org/10.36000/hbT.2020.01.002
}

healthbook TIMES Das Schweizer Ärztejournal Journal Des Médecins Suisse

Vol. 1, Issue 1, 2021

Coronavirus Disease 2019(COVID-19) has been widely spreading all over the world since the beginning of 2020. World Health Organization, on January $30^{\text {th }}$ 2020, declared the spread of COVID-19 to be a "public health emergency of international concern" (PHEIC). As of March 25 th $2020,414,179$ cases and 18,440 deaths due to COVID-19 had been reported worldwide. The disease is transmitted via inhalation or contact with infected droplets. The incubation period ranges from 2 to 14 days and can reach up to 21 days. Many people are asymptomatic. The estimated fatality rate ranges from $2 \%-3 \%$. Special molecular tests are being used to detect the virus in respiratory secretions. Elevated levels of C-reactive protein have been detected in the blood sample, while the white blood cell counts were considered normal. The computerized tomographic chest scan is usually abnormal even in those with no symptoms or mild disease. Current treatment options are essentially supportive, as the role of antiviral agents is yet to be established. Preventative measures can be utilized to slow the spread of infection, such as isolation of suspected cases or those with mild symptoms and strict infection control measures at hospitals. Though the virus is far more infectious than its two ancestors, severe acute respiratory syndrome coronavirus (SARS-CoV) and Middle East respiratory syndrome coronavirus (MERS-CoV), 2019-nCoV has a lower mortality rate. To date, the impact of this pandemic is uncertain, and the global research community is working diligently to find a satisfactory therapy. Several reports on Nigella sativa have been published, motivating us to review recent publications to summarize and investigate its possible therapeutic effect on the immune response and its potential to treat bacterial and viral infections, including COVID-19. For the advanced stage of the disease, we suggest using biological targeted magnetic hyperthermia. This can be used as adjuvant therapy, namely pseudo- adjuvant magnetic hyperthermia (PAMH).

\section{Introduction}

For centuries, various civilizations around the world have used Nigella sativa ( $N$. sativa) to treat ailments in both humans and animals. ${ }^{1}$ Numerous studies have been conducted on the therapeutic effects of the seed of $N$. sativa and

\footnotetext{
a Corresponding author:

Issa Rasheed Fetian, MD

FMH for General Internal Medicine,

HAP/MZB \& Diamon Institute,

University of Basel

Basel, Switzerland

E-mail: Irfetian@protonmail.com
} 
the main active constituent of its essential oil, thymoquinone (TQ). This is evidenced by the increasing number of published research reports. More than 406 research reports have been published about TQ from 1960 through 2014.

A number of pharmaceutical and biological properties have been ascribed to the seeds of $N$. sativa, such as antifungal, antibacterial, antiparasitic, and antiviral activity. ${ }^{1}$ TQ has shown efficacy against a variety of diseases, such as neurological and mental illnesses, cardiovascular disorders, cancer, diabetes, inflammatory conditions, and infertility. $N$. sativa has also recently gained increasing attention due to its strong antioxidant properties, leading to its frequent use as a dietary supplement. Additionally, TQ has demonstrated synergistic effects with various chemotherapeutic agents by optimizing efficacy and reducing toxicity.

Despite the limited data currently available, $N$. sativa has demonstrated promising efficacy against human immunodeficiency virus/acquired immunodeficiency syndrome (HIV/AIDS) and can be explored as an alternative option for the treatment of $2019-\mathrm{nCoV}^{1}$

\section{N. sativa potential therapeutic effects}

The antihypertensive effect of $N$. sativa appears to be mediated by a reduction in cardiac oxidative stress and angiotensin-converting enzyme activity, an increase in cardiac heme oxygenase- 1 activity, and prevention of plasma nitric oxide loss. Thus, $N$. sativa might be beneficial for controlling hypertension. ${ }^{2}$ It has an inhibitory effect of angiotensin-converting enzyme (ACE) that inhibits not only the conversion of angiotensin II from angiotensin I but also the bradykinase that degrades bradykinin. Bradykinin is generally a proinflammatory or an inflammatory mediator, which may inhibit viral replication.

In addition, the $N$. sativa has been reported to have significant activity against many diseased conditions, especially of the respiratory system such as asthma, bronchitis, and coughing. ${ }^{3}$ The bronchial relaxation effects of the boiled extract of $N$. sativa in contrast with theophylline were assessed in asthmatic patients, and it was found that the $N$. sativa extract caused substantial rises in the respiratory function tests and the starting time of bronchodilator action of the extract was comparable to that of theophylline. ${ }^{4} N$. sativa oils and active constituent (-hederin) also showed an improvement of tracheal responsiveness and significant anti-inflammatory activity via decreasing the release of histamine and leukotrienes while increasing the PGE2 from the mast cells and perfused lungs in an animal model of allergic asthma. 5,6 This antiasthmatic effect is further substantiated by different clinical studies, and most of them reported that different $N$. sativa preparations showed an improvement of clinical symptoms and pulmonary function as well as various asthma biomarkers. ${ }^{4,7,8}$ These preclinical and clinical studies evidenced the potential anti-asthmatic effects of $N$. sativa. 
It is clinically well-known that the upper and lower respiratory tract infections impair asthma and induce obstructive exacerbations. Exaggerated bronchial responsiveness in healthy subjects following upper respiratory infections was observed already 50 years ago. ${ }^{9}$ Viral infections are responsible for the deterioration in asthma in connection to respiratory infections as well as for the increased bronchial hyperreactivity observed during and following the infection. The mechanism for virus-induced bronchial hyper response could be explained and related to the airway pathology. The pathology after a virus infection shows many similarities to those observed in asthma. ${ }^{10} \mathrm{It}$ is, therefore, easy to imagine that virus infection can induce increased bronchial hyperresponsiveness and provoke asthma, with a multifactorial influence of the virus infection. There is support for epithelial cell damage as well as an immunological influence. ${ }^{10}$

For antibacterial activity, the antimicrobial properties of herbal plants and their extracts have been recognized since ancient times, while attempts to illustrate these qualities in the laboratory date back to the early 1900s. ${ }^{11}$ The surge of antibiotic-resistant germs highlights the necessity to find new antimicrobial agents to eradicate the infection and defeat the problems of resistance and side effects of the antimicrobial drugs that are currently in use. ${ }^{12,13}$ Extracts of $N$. sativa and specifically TQ exhibited broad antimicrobial range, including bacteria, viruses, and fungi. However, their effectiveness is dependent on the species of the target microorganisms. ${ }^{14}$ The mechanism of the antimicrobial effect of $N$. sativa seeds has not been reported; its antimicrobial property could be attributed to the active constituents, particularly TQ and melanin. ${ }^{15}$ Their broad spectrum of activity may be the reason that the key processes of the organisms are affected. ${ }^{16}$

In 2012, a study revealed that the combination of a half dose of ivermectin and $N$. sativa oil was more destructive to the cuticular or tegumental surfaces of adult helminths than each of them separately. The study had provided morphological evidence for the greater anthelmintic activity of ivermectin in combination with $N$. sativa oil, and the results lent support to the idea of using drug combinations against helminths infections. ${ }^{17}$

$N$. sativa possesses the antitubercular property. ${ }^{18} N$. sativa has excellent potential to be developed as a natural drug for the treatment of tuberculosis (TB), including drug-resistant TB. In a study conducted in 2013, it has been shown that the order of antitubercular activity of the tested quinonoids was plumbagin $>$ emodin $\sim$ menadione $\sim$ thymoquinone $>$ diospyrin, whereas their antibacterial efficacy was plumbagin $>$ menadione $\sim$ thymoquinone $>$ diospyrin $>$ emodin. Further investigations like infrared (IR) spectroscopy, nuclear magnetic resonance (NMR) and mass spectrometry (MS) are needed in order to determine the active metabolite. However, additional studies evaluating in vivo toxicity dosage and mechanism of action also need to be deciphered. The synergistic activities of purified compounds of $N$. sativa seeds 
with known TB drugs should be explored in the future as well. In light of the outbreak of COVID-19, it is a high necessity and priority to establish dynamic physiological studies that follow the development of the disease. This could be nuclear medicine, particularly positron emission tomography (PET), functional magnetic resonance imaging (fMRI), and computed tomography (CT) images. The fusion of different modalities is to be recruited to get better details for structure and function. Machine learning and AI studies are highly recommended. This aims to get a differential diagnosis with high confidence of COVID-19 symptoms to differentiate it from other most common symptoms.

Recent publications have brought attention to possible benefits of chloroquine, a broadly used antimalarial drug, in the treatment of patients who are infected by the novel emerging SARS-CoV-2. ${ }^{19}$ The scientific community has considered this information in light of previous experiments with chloroquine in the field of antiviral research. On the other hand, the N. sativa was given to the patients who were resistant to different antimalarial therapies as well as antiviral therapies, but in addition, it is now broadly used in autoimmune diseases such as rheumatoid arthritis. Comparing this drug to others, it is considered to be safe with generally transitory side-effects.

Endothelins are potent regulators of vascular tone, which also have mitogenic, apoptotic, and immunomodulatory properties. ${ }^{20,21}$ Three isoforms of endothelin have been identified to date, with endothelin-1 (ET-1) being considered the best route of study. ET-1 is classically considered a potent vasoconstrictor. However, in addition to the effects of ET-1 on vascular smooth muscle cells, the peptide is increasingly recognized as a proinflammatory cytokine. ${ }^{21,22}$ ET-1 causes platelet aggregation and plays a role in the increased expression of leukocyte adhesion molecules, the synthesis of inflammatory mediators contributing to vascular dysfunction. High levels of ET-1 are found in alveolar macrophages, leukocytes, and fibroblasts. ${ }^{22,23}$ Clinical and experimental data indicate that ET-1 is involved in the pathogenesis of sepsis, ${ }^{24,25}$ viral and bacterial pneumonia, ${ }^{26,27}$ Rickettsia conorii infections, ${ }^{28}$ Chagas disease, ${ }^{29,30}$ and severe malaria. ${ }^{31,32}$ A postmortem histological analysis study of COVID-19 patients in Zurich revealed viral inclusion structures in endothelial cells. This histological study showed endotheliitis in many organs of the above-mentioned patients. In addition to that, endothelial dysfunction is a principal determinant of microvascular dysfunction by shifting the vascular equilibrium towards more vasoconstriction with subsequent organ ischemia, inflammation with associated tissue edema, and procoagulant state. ${ }^{33} \mathrm{~A}$ study has explained by their findings that the presence of viral elements within endothelial cells and an accumulation of inflammatory cells, with evidence of endothelial and inflammatory cell death. These findings suggest that SARS-CoV-2 infection facilitates the induction of endotheliitis in several organs as a direct consequence of viral involvement and of the host inflammatory response (Figure 1). In addition, induction of apoptosis and pyroptosis might have 

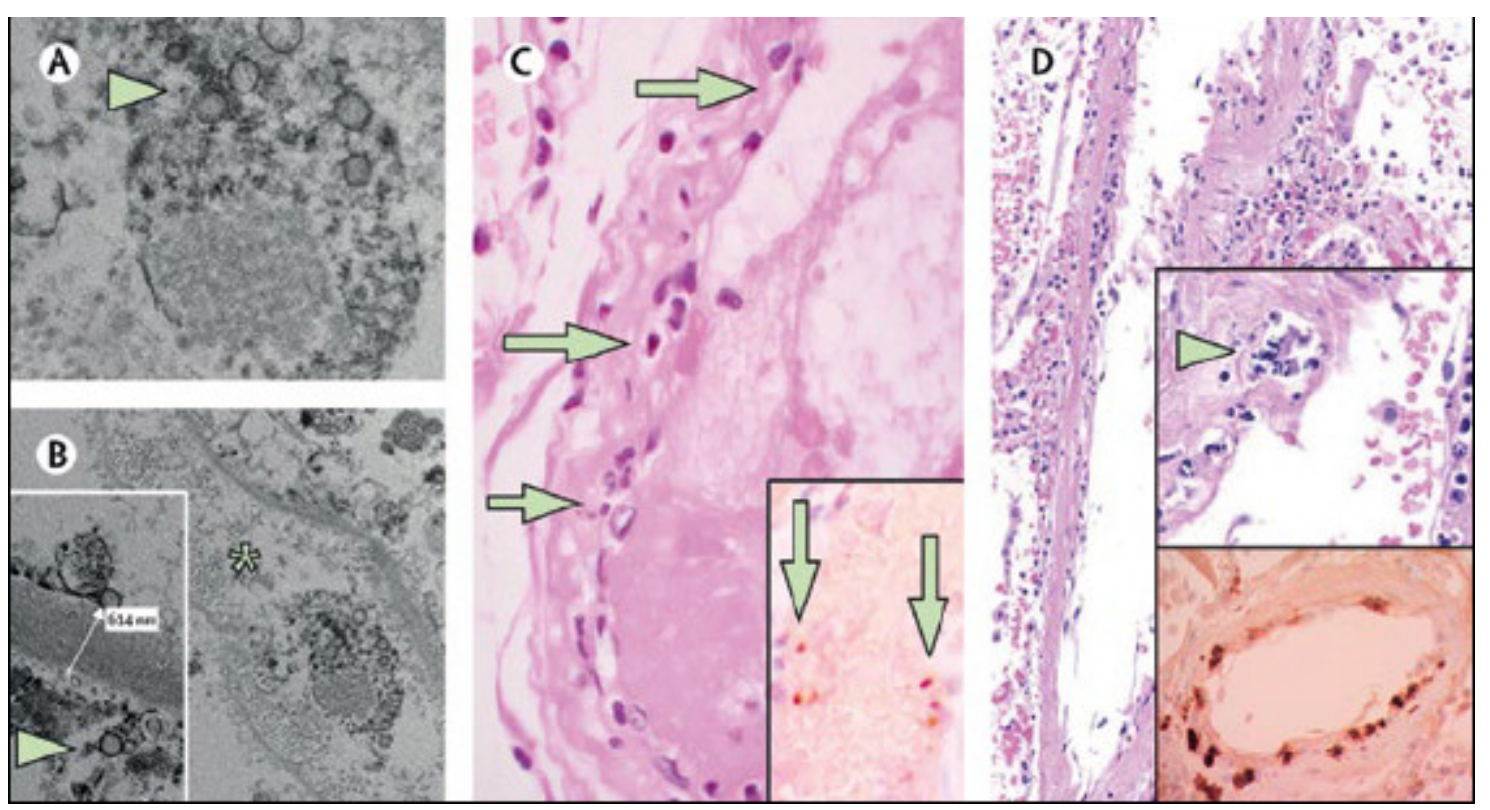

Figure 1. Endotheliitis.

Adapted from Varga et al. 2020. ${ }^{34}$ (A), (B) Electron microscopy of kidney tissue shows viral inclusion bodies in a peritubular space and viral particles in endothelial cells of the glomerular capillary loops. Aggregates of viral particles (arrow) appear with dense circular surface and lucid center. The asterisk in panel B marks peritubular space consistent with capillary containing viral particles. The inset in panel B shows the glomerular basement membrane with endothelial cell and a viral particle (arrow; about $150 \mathrm{~nm}$ in diameter). (C) Small bowel resection specimen of patient 3, stained with haematoxylin and eosin. Arrows point to dominant mononuclear cell infiltrates within the intima along the lumen of many vessels. The inset of panel C shows an immunohistochemical staining of caspase 3 in small bowel specimens from serial section of tissue described in panel D. Staining patterns were consistent with apoptosis of endothelial cells and mononuclear cells observed in the haematoxylin-eosin-stained sections, indicating that apoptosis is induced in a substantial proportion of these cells. (D) Post-mortem lung specimen stained with haematoxylin and eosin showed thickened lung septa, including a large arterial vessel with mononuclear and neutrophilic infiltration (arrow in upper inset). The lower inset shows an immunohistochemical staining of caspase 3 on the same lung specimen; these staining patterns were consistent with apoptosis of endothelial cells and mononuclear cells observed in the haematoxylin-eosin-stained sections.

an important role in endothelial cell injury in patients with COVID-19. ${ }^{34} \mathrm{~N}$. sativa has a reducing effect on oxidative-like endothelin and it reduces the endothelin level in bronchoalveolar lavage and lung tissue. ${ }^{35}$

Nitric oxide (NO) is considered to be a broncho- and vasodilator, which is synthesized from L-arginine by nitric oxide synthases (NOS). ${ }^{36} \mathrm{NO}$ deficiency results in airway hyperreactivity and endothelial dysfunction. ${ }^{37,38}$ Consequently leading to an increase in NO production. ${ }^{39}$ It is known that pulmonary obstruction increases NO levels, which lead to higher production of the NO-inhibiting metabolites, such as asymmetric dimethylarginine (ADMA) and symmetric dimethylarginine (SDMA). ${ }^{40}$

ADMA and SDMA are derived from the proteolysis of these methylated arginines on various proteins. They also showed an association with disease severity as assessed with the pneumonia severity index. ${ }^{41} N$. sativa has been reported to contain amino acids and vitamin supplements. ${ }^{42}$ Raising arginine levels by supplementing this amino acid in critically ill patients showed controversial results. ${ }^{43}$ In 2014 a study also has shown that administration 
of L-arginine enhanced the anti-inflammatory activity of $N$. sativa oil. ${ }^{44}$ It is believed that taking NS will raise the arginine level, which accelerates the antiinflammatory effect which improves the endothelial cell function.

Factor $\mathrm{Xa}$ is a serine proteinase that functions principally at coagulation cascades. Factor Xa-like immunoreactivity has been examined in several human organs. Furthermore, the preferential localization to the epithelium in the nose and bronchus is interesting in view of the previous notion that several viruses targeting the respiratory tract require factor Xa-like cellular proteases for their replication and spread. ${ }^{45} \mathrm{~A}$ study published in the Journal of Molecular Science has shown that TQ, both extracted from black seeds and its pure form, has the ability to modulate blood coagulation in vitro. It was confirmed via mechanistic studies that TQ interferes with the blood coagulation by directly decreasing, weakly, but significantly, the activity of factor $\mathrm{Xa}$ in blood coagulation pathway and by down-regulating the downstream effects of TNF$\alpha$, a cytokine that plays a critical role in the crosstalk between the inflammatory pathway and thrombosis. ${ }^{46}$ Interestingly, the methanol soluble portion of black cumin oil, which is prepared by compression of $N$. sativa seeds, showed inhibitory effects on arachidonic acid (AA)-induced platelet aggregation and blood coagulation. Compounds possessing aromatic hydroxyl and acetoxyl group had more potent activity than aspirin, which is well-known as a remedy for thrombosis. ${ }^{47}$

It has been reported that apoptosis is programmed cell death in response to pathological or physiological changes, which are directed to eliminate the dead, mutated, or aged cell. Apoptosis is caused by viral infections leading to lymphocyte depletion in the host cell, and antioxidants can inhibit apoptosis, which is induced by viruses and can stop the viral replication in target cells. Thus, antiviral and antioxidant effects can be linked together. ${ }^{48}$ The antiviral effect of the $N$. sativa is related to the increasing response of CD4 cells. ${ }^{49} \mathrm{In}$ a research paper, the patient with hepatitis $\mathrm{C}$ virus $(\mathrm{HCV})$ infection, who was not eligible for IFN- $\alpha$ therapy, had received $N$. sativa oil (NSO) capsule (450 $\mathrm{mg}$ ) three times a day for three consecutive months, displayed a significant decrease in viral load and improvement of the oxidative stress due to augmented total antioxidant activity, total protein and albumin as well as improved red blood cell (RBC) and platelet counts. The augmented RBC count can be attributed to the lowering of the membrane lipid peroxide level, which leads to reduced incidence of hemolysis. ${ }^{50}$ By diminishing the blood glucose levels, it may provide a potential modulatory influence on $\mathrm{HCV}$ induced glucose intolerance. Furthermore, improvement in the lower limb edema also was seen. ${ }^{50}$

Recently, it was proven that the thymoquinone activity efficiently inhibits the survival of Epstein-Barr virus (EBV)-infected B cells and alters EBV gene expression, which was explained through the high potential to induce apoptosis in EBV infected cells. ${ }^{51}$ TQ has not been reported to cause 
suppression of bone marrow function. ${ }^{52}$ Ulasli et al. combined $N$. sativa extracts with the ones before infection with SARS-CoV and virus replication was decreased accordingly. ${ }^{53}$

On the other hand, $N$. sativa plays a vital role in insulin secretion by improving energy metabolism in mitochondria and other intracellular pathways of insulin receptors. ${ }^{54}$ It can participate in rising insulin concentration and plays a role in the inhibition of the hypothalamus-pituitary-adrenal-axis, which leads to better insulin effects. Protection against infectious challenges is the primary function of the immune system. In response to viral infection, adaptive immunity is required to limit the initial spread of the virus and ultimately terminate viral replication. Proinflammatory cytokines induced during viral infection activate the hypothalamic-pituitary-adrenal axis. Activation of the hypothalamic-pituitary-adrenal axis is a reflection of the fact that the immune system is functionally integrated with the nervous and endocrine systems. ${ }^{55}$ This alteration of insulin secretion, considered to be a stress factor in addition to the viral infection, will activate the hypothalamic-pituitary-adrenal axis, which plays an important immunomodulatory role with the elevation of plasma levels of glucocorticoid hormones with ability to mediate adaptive behavioral, metabolic, cardiovascular, and immune system effects. In combination with immobilization and pronounced inflammatory and endocrine stress response, these contribute to muscle wasting and progressive deterioration of metabolic and functional status, particularly in patients with multiple morbidities. ${ }^{56}$

Mitochondria are the only intracellular organelles whose interior is negatively charged relative to the exterior. ${ }^{57,58}$ This fact can be used to address various compound physiological processes specifically. To this end, it was suggested to combine the transported compound with a positively charged ion easily penetrating through biomembranes. To make an ion permeable for membranes, its ionized atom should be surrounded by bulky hydrophobic residues that delocalize the electric charge of this atom. ${ }^{57,59,60}$ Such a principle was employed to construct mitochondria-targeted antioxidants. ${ }^{60,61}$ Among them, some quinone derivatives proved to be the most active.

Data provided evidence that miRNA-mediated regulation of cytochrome $c$ oxidase (COX6C) mRNA expression in primary human bronchial epithelial cells and lung epithelial cells leads to apoptosis in the early stage of infection. ${ }^{62}$ COX6C is the last enzyme in the respiratory electron transport chain (ETC) of mitochondria. Its main function is to convert molecular oxygen to water and aid in establishing mitochondrial membrane potential. The data demonstrated that low expression of COX6C is highly favorable for viral replication, possibly through reduced and longer survival of the infected cell. Decreased COX6C resulted in decreased caspase- 9 protein release and eventually led to increased viral copy number. 
COX6C is usually required by the host cell for the induction of the apoptotic signaling pathway and the reduction of viral numbers. According to a study in $2019, N$. sativa inhibits the deactivated function of mitochondria induced by oxidative stress as indicated by retrieving cytochrome c oxidase activity in rats. 63

$N$. sativa seems to be the right drug for treating COVID-19 and different viral infections, but until now, no clinical trial has tested the effects as the first-line drug for the treatment of the new disease.

On the other hand, it can be combined with other adjuvant therapies. Hyperthermia is a case in point. Hyperthermia refers to a therapeutic modality by which a given region of interest is subjected to temperature $(\mathrm{T})$ increased above $40 \circ C .64,65$ It is reported that Hippocrates stated: "Those who cannot be cured by medicine can be cured by surgery. Those who cannot be cured by surgery can be cured by fire." (hyperthermia). "Those who cannot be treated by fire cannot be treated at all.”. Hyperthermia has been utilized in several randomized trials in cancer treatment demonstrating that when combined with radiotherapy, it has the potential to improve the outcomes of various cancers without increasing toxicity. 66,67

The major shortcoming of conventional hyperthermia is that basically, both healthy and abnormal cells (e.g., malignant cells), have equal biological response sensitivity to heat. ${ }^{68}$ To overcome this problem, a significant interest came to the horizon for relatively new hyperthermia modality - the magnetic hyperthermia $(\mathrm{MH})$ or the concept of "biologically targeted magnetic hyperthermia. Targeted magnetic iron oxide nanoparticles (MION) are injected intravenously as a pro heating via an alternating magnetic field (AMF).

It is suggested here to use the MION concept to help manage the breakout of COVID-19 and in combination with $N$. sativa. We name this as pseudoadjuvant magnetic hyperthermia (PAMH).

SARS-CoV-2 has been shown to bind to angiotensin-converting enzyme 2 (ACE 2) to enter human cells by binding via the $S$ protein on its surface. During infection, the $S$ protein is cleaved into subunits $S 1$ and S2. The $S 1$ contains the receptor-binding domain (RBD), which allows coronaviruses to directly bind to the peptidase domain (PD) of ACE 2, and the S2 is believed to have a significant role in membrane fusion. ${ }^{69}$ Through the understanding of the ACE 2, it is expected to lead to producing antivirus or a vaccine that can block coronavirus infection by targeting ACE 2.

Hyperthermia can have adverse impacts on the cytoskeleton, organelles, intracellular transport, and RNA processing. ${ }^{70}$ 


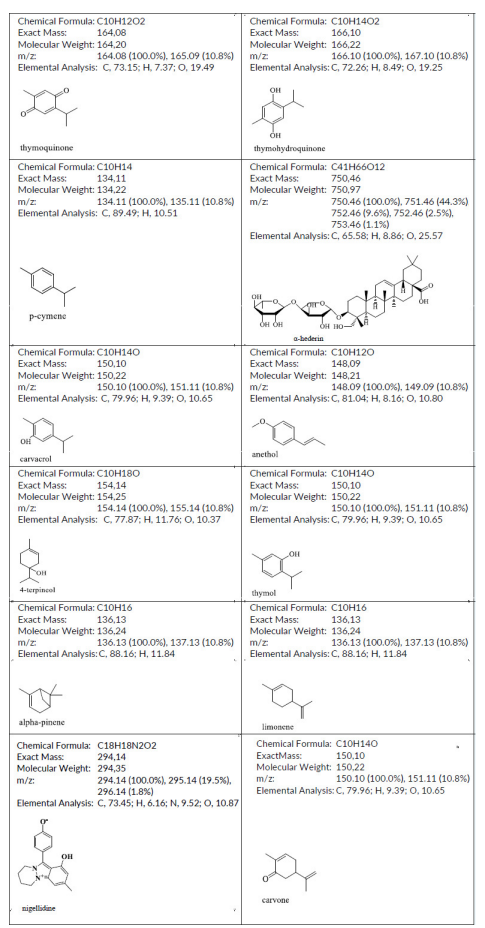

Table 1. Active components of Nigella sativa.

Using PAMH has the potential of blocking the ACE 2 from binding to $S$ protein and by the adverse impact on virus RNA. In addition, the elevated temperature is combined with NS unique pharmacological properties in boosting the immune system. It is a promising combination in managing the breakout of the COVID-19.

\section{Chemical Composition and the Dosage Forms}

Several complex compounds have been extracted and studied from the $N$. sativa seeds. Thymoquinone (TQ) is one of the most abundant components in the seed's essential oil (Table 1). It constitutes about 30-48\% depending on the variety of the seeds. Other components include, thymohydroquinone (THQ), dithymoquinone, p-cymene, carvacrol, - -hederin, 4-terpineol, thymol, tanethol, $\alpha$-pinene, limonene, nigellidine, and sesquiterpene longifolene. Additionally, the seeds are reported to contain several vitamins, and minerals like $\mathrm{Cu}, \mathrm{P}, \mathrm{Zn}, \mathrm{Fe}$ and carotene, which is converted by the liver to vitamin A. ${ }^{42,71}$ It is of note that the N. sativa affects the anti-proliferative activity on human cancer and normal cells. FTIR and UV-Vis spectrometry showed selective anti-proliferation activity against estrogen-dependent on human breast cancer cells at $60^{\circ} \mathrm{C}$ and 2500 psi. $^{72}$

Combination of 5-fluorouracil and TQ potentiate the cytotoxic effects of the individual compounds and simultaneously target a broader spectrum of oncogenic pathways leading to an effective eradication of colorectal cancer cells. $^{73}$ 
Thymoquinone-artemisinin hybrids were found to have an efficient and broad variety of biological activities combined with a low toxicity and high selectivity profile. $^{74}$

Most of the medicinal properties of $N$. sativa were explored as an orally provided nutritional or dietary supplement of ground seeds in tablet or capsule form, oil extract in soft gel capsules, or as an aqueous extract in the form of fluid. ${ }^{75}$ Different combinations of $N$. sativa powder or extract with other vitamins such as vitamin $\mathrm{E}$ are commonly sold as black seeds extract or black cumin oil with claims of enhancing the body's natural defense or promoting healthier skin as an antioxidant.

\section{Bioavailability}

It is known that the bioavailability of any therapeutic agent is an important factor to achieve therapeutic efficacy. ${ }^{76}$ It is also known that solubility is one of the most physicochemical properties that proportionally impact the bioavailability of drugs.

Various nanoformulation techniques such as liposomes, solid lipid nanoparticles (SLNs), niosomes, nanostructured lipid carriers (NLCs), and nanoemulsions have been explored to enhance the bioavailability and the efficacy of TQ.

When the self-nano emulsifying drug delivery system of TQ was prepared, its bioavailability has been increased 3.87 -fold in comparison to TQ suspension. ${ }^{77-80}$ The bioavailability of TQ has been reported to get enhanced 5 times by SLN preparation in comparison to conventional formulations when tested on albino Wistar rats. ${ }^{81}$

In an in vivo study performed on albino Wistar rats, TQ shows enhanced bioavailability in NLC formulation when compared to suspension by 3.97 -fold with increased plasma half-life. ${ }^{82}$

\section{Safety and Toxicity}

Toxicity studies play an important role in the safety assessment of new therapeutic entities prior to human trials application. Several animal models have been proven to be predictive of toxicity effects on humans.

The LD50 (median lethal dose) of TQ, when administered in mice were found to be $870.9 \mathrm{mg} / \mathrm{kg}$ and $104.7 \mathrm{mg} / \mathrm{kg}$ after oral and intraperitoneal (IP) route of administration, respectively. Similarly, the LD50s in rats were $794.3 \mathrm{mg} /$ $\mathrm{kg}$ and $57.5 \mathrm{mg} / \mathrm{kg}$ after oral and IP administration, respectively. ${ }^{77,83,84}$ The toxicity signs after oral administration of TQ reported difficulties in respiration (dyspnea) and peritonitis after injection in rats and mice. ${ }^{85}$ However, it is also reported that after long term administration of TQ alone or TQ-NLC causes 
liver toxicity but at the tolerable dose, it does not affect the organ function. Few authors also concluded that the NLC formulation of TQ increased the tolerability of TQ alone in their experiment up to $100 \mathrm{mg} / \mathrm{kg} .{ }^{85,86}$

The nephroprotective effects of TQ in different pathogenic conditions have been reported. TQ was found to enhance the appearance of renal lesions resulting from various toxic agents partly by weakening reactive oxygen species and inflammation. TQ treatment ameliorated acute renal failure induced by gentamicin in rats by restoring mitochondrial function and salvaging ATP production. ${ }^{87}$ In the same study, it has been shown that it improves renal function markers such as blood urea nitrogen and creatinine. ${ }^{88}$

\section{Future Aspects}

Ideally, a clinical study with a subgroup of patients with COVID-19 can be well characterized. Supplementing with the composition like in N. Sativa will improve the general health of the patients. The scientific community should consider this information in light of previous experiments with $N$. sativa in the field of antiviral research.

\section{Conclusion}

$N$. sativa has unique properties that made it distinguished as a pharmacological drug. So many studies conducted since 1959 confirmed that $N$. sativa has a complex structure of more than 100 compounds, some of which have not yet been identified nor studied. The unique combination of these complex structures of volatile oils and other trace elements is believed to play a major role in $N$. sativa therapeutic effectiveness. The original research articles published so far have shown the potential immunomodulatory and immunotherapeutic potentials of $N$. sativa active ingredients, in particular thymoquinone (TQ). The immunotherapeutic efficacy of TQ is linked to its antitoxic, antihistamine, immunomodulatory, and anti-inflammatory properties.

The immunotherapeutic efficacy of ingestion or administration of the whole seeds, oil, or its purified constituents should be measured by the nature of the disease.

Therefore, further studies are required to explore the specific cellular and molecular targets of $N$. sativa constituents in particular TQ. TQ immunomodulation properties and its other health benefits exhibit promising potential to be used as a drug to prevent or treat the viral infection caused by COVID-19.

\section{TAKE-HOME MESSAGES}

- Prevention and case management of $\mathrm{n}-\mathrm{CoV} 19$ poses one of the greatest challenges to the current situation. 
- Within $N$. sativa there are structural compounds that exhibit immunomodulatory effects, which will be harnessed towards nCoV19 management.

- The main active constituent of $N$. sativa "TQ" demonstrates a therapeutic effect on immune-response with great potential to treat bacterial and viral infections.

- It could also be used against many of the respiratory system symptoms and conditions like asthma, bronchitis, and coughing.

- Oxidizing the iron component of $N$. sativa and using the fluid form enables pro heating and circulation via blood flow to the viral site destination.

- Using PAMH has the potential of blocking the ACE 2 from binding to $S$ protein and by the adverse impact on virus RNA.

\section{Autbors' contributions}

All authors participated in the conception, data collection, and data analysis and writing the manuscript critical review and making revisions. All authors read and approved the manuscript.

\section{Funding}

None.

\section{Acknowledgments}

This work was not supported by any fund or organization. It is an authors' initiative project funded exclusively from their personal sources.

\section{Conflict of interest}

The authors declare that the research was conducted in the absence of any commercial or financial relationships that could be construed as a potential conflict of interest.

\section{Consent for publication}

Not applicable. 


\section{REFERENCES}

1. Yimer EM, Tuem KB, Karim A, Ur-Rehman N, Anwar F. Nigella sativa L. (Black Cumin): A Promising Natural Remedy for Wide Range of Illnesses. Evid Based Complementary Altern Med. Published online 2019:1-16. doi:10.1155/2019/1528635

2. Jaarin K, Foong WD, Yeoh MH, et al. Mechanisms of the antihypertensive effects of Nigella sativa oil in L-NAME-induced hypertensive rats. Clinics (Sao Paulo). 2015;70(11):751-757. doi:10.6061/clinics/2015(11)07

3. Rajsekhar $S$ et al. Pharmacognosy and pharmacology of Nigella sativa-A review. Int Res J Pharm. 2011;2(11):36-39.

4. Boskabady MH, Mohsenpoor N, Takaloo L. Antiasthmatic effect of Nigella sativa in airways of asthmatic patients. Phytomedicine. 2010;17(10):707-713. doi:10.1016/j.phymed.2010.01.002

5. Ikhsan M, Hiedayati N, Maeyama K, Nurwidya F. Nigella sativa as an anti-inflammatory agent in asthma. BMC Res Notes. 2018;11(1):744. doi:10.1186/s13104-018-3858-8

6. Boskabady MH, Keyhanmanesh R, Khamneh S, Ebrahimi MA. The effect of Nigella sativa extract on tracheal responsiveness and lung inflammation in ovalbumin-sensitized guinea pigs. Clinics (Sao Paulo). 2011;66(5):879-887. doi:10.1590/s1807-59322011000500027

7. Salem AM, Bamosa AO, Qutub HO, et al. Effect of Nigella sativa supplementation on lung function and inflammatory mediatorsin partly controlled asthma: a randomized controlled trial. Ann Saudi Med. 2017;37(1):64-71. doi:10.5144/0256-4947.2017.64

8. Boskabady MH, Farhadi J. The Possible Prophylactic Effect of Nigella sativa Seed Aqueous Extract on Respiratory Symptoms and Pulmonary Function Tests on Chemical War Victims: A Randomized, Double-Blind, Placebo-Controlled Trial.J Altern Complement Med.

2008;14(9):1137-1144. doi:10.1089/acm.2008.0049

9. Parker CD. Methacholine Aerosol As Test For Bronchial Asthma. Arch Intern Med. 1965;115(4):452. doi:10.1001/archinte.1965.03860160078013

10. Wennergren G. Impact of viral infection on bronchial hyperresponsiveness. Pediatr Allergy Immunol. 1996;7(9 Suppl):10-13. doi:10.1111/j.1399-3038.1996.tb00387.x

11. Dorman HJD, Deans SG. Antimicrobial agents from plants: antibacterial activity of plant volatile oils. J Appl Microbiol. 2000;88(2):308-316. doi:10.1046/j.1365-2672.2000.00969.x

12. Morsi NM. Antimicrobial effect of crude extracts of Nigella sativa on multiple antibioticsresistant bacteria. Acta Microbiol Pol. 2000;49(1):63-74.

13. Hannan A, Saleem S, Chaudhary S, Barkaat M, Arshad MU. Anti bacterial activity of Nigella sativa against clinical isolates of methicillin resistant Staphylococcus aureus.J Ayub Med Coll Abbottabad. 2008;20(3):72-74.

14. Forouzanfar F, Bazzaz BSF, Hosseinzadeh H. Black cumin (Nigella sativa) and its constituent (thymoquinone): a review on antimicrobial effects. Iran J Basic Med Sci. 2014;17(12):929-938.

15. Bakathir HA, Abbas NA. Detection of the antibacterial effect of Nigella sativa ground seedswith water. Afr J Trad Compl Alt Med.2011;8(2). doi:10.4314/ajtcam.v8i2.63203

16. Monika T et al. An investigational study of antibacterial activities of Nigella sativa on Mastits in dairy crossbred cows. Int J Adv Scient Tech Res. 2013;3:273-283.

17. Sayed-Ahmed MM, Nagi MN. Thymoquinone supplementation prevents the development of gentamicin-induced acute renal toxicity in rats. Clin Exp Pharmacol Physiol. 2007;34(5-6):399-405. doi:10.1111/j.1440-1681.2007.04560.x

18. Anbarasu S, et al. Evaluating the Anti-mycobacterial Activity of Nigella sativa Seed Extracts. Der Pharm Lett. 2018;10(7):1-9. 
19. Colson P, Rolain JM, Raoult D. Chloroquine for the 2019 novel coronavirus SARS-CoV-2. Int J Antimicrob Agents. 2020;55(3):105923. doi:10.1016/j.jjantimicag.2020.105923

20. Kedzierski RM, Yanagisawa M. Endothelin system: the double-edged sword in health and disease. Annu Rev Pharmacol Toxicol. 2001;41(1):851-876. doi:10.1146/ annurev.pharmtox.41.1.851

21. Bagnato A, Loizidou M, Pflug B, Curwen J, Growcott J. Role of the endothelin axis and its antagonists in the treatment of cancer. Br J Pharmacol.2011;163(2):220-233. doi:10.1111/ j.1476-5381.2011.01217.x

22. Sessa WC, Kaw S, Hecker M, Vane JR. The biosynthesis of endothelin-1 by human polymorphonuclear leukocytes. Biochem Biophys Res Commun. 1991;174(2):613-618. doi:10.1016/ $\underline{0006-291 X(91) 91461-K}$

23. Gu J, et al. Detection of endothelin-like immunoreactivity in epithelium and fibroblasts of the human umbilical cord. Tissue Cell. 1991;2(34):237-244.

24. Tschaikowsky K, Sđner S, Lehnert N, Kaul M, Ritter J. Endothelin in septic patients: Effects on cardiovascular and renal function and its relationship to proinflammatory cytokines: Crit Care Med. 2000;28(6):1854-1860. doi:10.1097/00003246-200006000-00028

25. Goto T, Hussein MH, Kato S, et al. Endothelin receptor antagonist attenuates oxidative stress in a neonatal sepsis piglet model. Pediatr Res. 2012;72(6):600-605. doi:10.1038/pr.2012.134 26. Schuetz P, Stolz D, Mueller B, et al. Endothelin-1 precursor peptides correlate with severity of disease and outcome in patients with community acquired pneumonia. BMC Infect Dis. 2008;8(1):22. doi:10.1186/1471-2334-8-22

27. Samransamruajkit $\mathrm{R}$ et al. Plasma endothelin-1 in infants and young children with acute bronchiolitis and viral pneumonia. Asian Pac J Allergy Immunol. 2002;20(4):229.

28. Davì $G$ et al. Demonstration of Rickettsia Conorii-induced coagulative and platelet activation in vivo in patients with Mediterranean spotted fever. Thromb Haemost. 1995;74(2):631-634.

29. Petkova SB, Huang H, Factor SM, et al. The role of endothelin in the pathogenesis of Chagas' disease. Int J Parasitol. 2001;31(5-6):499-511. doi:10.1016/S0020-7519(01)00168-0

30. Petkova SB, Tanowitz HB, Magazine HI, et al. Myocardial Expression of Endothelin-1 in Murine Trypanosoma cruzi Infection. Cardiovasc Pathol. 2000;9(5):257-265. doi:10.1016/ $\underline{\text { S1054-8807(00)00045-4 }}$

31. Dai M, Freeman B, Bruno FP, et al. The novel ETA receptor antagonist HJP-272 prevents cerebral microvascular hemorrhage in cerebral malaria and synergistically improves survival in combination with an artemisinin derivative. Life Sci. 2012;91(13-14):687-692. doi:10.1016/ j.lfs.2012.07.006

32. Dietmann A, Lackner P, Helbok R, et al. Opposed circulating plasma levels of endothelin-1 and C-type natriuretic peptide in children with Plasmodium falciparum malaria. MalarJ. 2008;7(1):253. doi:10.1186/1475-2875-7-253

33. Bonetti PO, Lerman LO, Lerman A. Endothelial Dysfunction: A Marker of Atherosclerotic Risk. Arterioscler Thromb Vasc Biol. 2003;23(2):168-175. doi:10.1161/ 01.ATV.0000051384.43104.FC

34. Varga Z, Flammer AJ, Steiger P, et al. Endothelial cell infection and endotheliitis in COVID-19. Lancet. 2020;395(10234):1417-1418. doi:10.1016/S0140-6736(20)30937-5

35. Mazouchian $\mathrm{H}$ et al. The effect of thymoquinone, the main constituent of Nigella sativa, on endothelin level of ovalbumin sensitized guinea pigs. Adv Biores. 2013;4(5):105-108. 
36. Nickler M, Ottiger M, Steuer C, et al. Systematic review regarding metabolic profiling for improved pathophysiological understanding of disease and outcome prediction in respiratory infections. Respir Res. 2015;16(1):125. doi:10.1186/s12931-015-0283-6

37. Scott J, Duongh M, Young A, Subbarao P, Gauvreau G, Grasemann H. Asymmetric Dimethylarginine in Chronic Obstructive Pulmonary Disease (ADMA in COPD). Int J Mol Sci. 2014;15(4):6062-6071. doi:10.3390/ijms15046062

38. Shivkar RR. Ratio Of Serum Asymmetric Dimethyl Arginine (ADMA)/ Nitric Oxide in Coronary Artery Disease patients.J Clin Diagn Res. 2014;8(8):CC04. doi:10.7860/JCDR/2014/ $\underline{7849.4665}$

39. Epstein FH, Moncada S, Higgs A. The L-Arginine-Nitric Oxide Pathway. N Engl J Med. 1993;329(27):2002-2012. doi:10.1056/NEJM199312303292706

40. Ruzsics I, Nagy L, Keki S, et al. L-Arginine Pathway in COPD Patients with Acute Exacerbation: A New Potential Biomarker. COPD. 2016;13(2):139-145. doi:10.3109/ $\underline{15412555.2015 .1045973}$

41. Vögeli A, Ottiger M, Meier MA, et al. Admission levels of asymmetric and symmetric dimethylarginine predict long-term outcome in patients with community-acquired pneumonia. Respir Res. 2017;18(1):25. doi:10.1186/s12931-017-0502-4

42. Al-Jassir MS. Chemical composition and microflora of black cumin (Nigella sativa L.) seeds growing in Saudi Arabia. Food Chem. 1992;45(4):239-242. doi:10.1016/0308-8146(92)90153-S

43. Visser M, Vermeulen MAR, Richir MC, et al. Imbalance of arginine and asymmetric dimethylarginine is associated with markers of circulatory failure, organ failure and mortality in shock patients. BrJ Nutr. 2012;107(10):1458-1465. doi:10.1017/S0007114511004648

44. Amin A et al. Effect of Nigella sativa (Blackseed) oil on inflammation and nitric oxide productionin wistar rats. 2014;3:112-113.

45. Yamada $T$ et al. Immunohistochemical studies of human tissues with antibody to factor Xa.J Histochem. 1996;28(1):73-77.

46. Muralidharan-Chari V, Kim J, Abuawad A, Naeem M, Cui H, Mousa S. Thymoquinone modulates blood coagulation in vitro via its effects on inflammatory and coagulation pathways. Int J Mol Sci. 2016;17(4):474. doi:10.3390/ijms17040474

47. Enomoto S, Asano R, Iwahori Y, et al. Hematological Studies on Black Cumin Oil from the Seeds of Nigella sativa L. Biol Pharm Bull. 2001;24(3):307-310. doi:10.1248/bpb.24.307

48. Peterhans E. Oxidants and Antioxidants in Viral Diseases: Disease Mechanisms and Metabolic Regulation. NutrJ. 1997;127(5):962S-5S. doi:10.1093/jn/127.5.962S

49. Salem ML, Hossain MS. Protective effect of black seed oil from Nigella sativa against murine cytomegalovirus infection. Int Immunopharmacol. 2000;22(9):729-740. doi:10.1016/ $\underline{\text { S0192-0561(00)00036-9 }}$

50. Barakat EMF. Effects of Nigella sativa on outcome of hepatitis C in Egypt. WorldJ Gastroenterol. 2013;19(16):2529. doi:10.3748/wjg.v19.i16.2529

51. Zihlif MA, Mahmoud IS, Ghanim MT, et al. Thymoquinone Efficiently Inhibits the Survival of EBV-Infected B Cells and Alters EBV Gene Expression. Integr Cancer Ther. 2013;12(3):257-263. doi: $10.1177 / 1534735412458827$

52. Gali-Muhtasib H, Roessner A, Schneider-Stock R. Thymoquinone: A promising anti-cancer drug from natural sources. Int J Biochem Cell Biol. 2006;38(8):1249-1253. doi:10.1016/ j.biocel.2005.10.009 
53. Ulasli M, Gurses SA, Bayraktar R, et al. The effects of Nigella sativa (Ns), Anthemis hyalina $(\mathrm{Ah})$ and Citrus sinensis (Cs) extracts on the replication of coronavirus and the expression of TRP genes family. Mol Biol Rep. 2014;41(3):1703-1711. doi:10.1007/s11033-014-3019-7

54. Mansi KS. Effects of oral administration of water extract of Nigella sativa on the hypothalamus pituitary adrenal axis in experimental diabetes. Int J Pharmacol. 2006;2(1):104-109.

55. McEwen BS, Biron CA, Brunson KW, et al. The role of adrenocorticoids as modulators of immune function in health and disease: neural, endocrine and immune interactions. Brain Res Rev. 1997;23(1-2):79-133. doi:10.1016/S0165-0173(96)00012-4

56. Felder S, Braun N, Stanga Z, et al. Unraveling the Link between Malnutrition and Adverse Clinical Outcomes: Association of Acute and Chronic Malnutrition Measures with Blood Biomarkers from Different Pathophysiological States. Ann Nutr Metab. 2016;68(3):164-172. doi: $10.1159 / 000444096$

57. Liberman EA, Skulachev VP. Conversion of biomembrane-produced energy into electric form. IV. General discussion. Biochim Biophys Acta Biomembr. 1970;216(1):30-42. doi:10.1016/ $\underline{0005-2728(70) 90156-8}$

58. Skulachev VP et al. Membrane Bioenergetics. Springer-Verlag; 1988.

59. Liberman EA, Topaly VP, Tsofina LM, Jasaitis AA, Skulachev VP. Mechanism of coupling of ocxidative phosphorylation and the membrane potential of mitochondria. Nature.

1969;222(5198):1076-1078. doi:10.1038/2221076a0

60. Trendeleva TA, Sukhanova EI, Rogov AG, et al. Role of charge screening and delocalization for lipophilic cation permeability of model and mitochondrial membranes. Mitochondrion .

2013;13(5):500-506. doi:10.1016/j.mito.2012.10.006

61. Skulachev V P et al. Principles of Bioenergetics. Springer-Verlag; 2013. doi:10.1007/ 978-3-642-33430-6

62. Othumpangat $S$, Noti JD, Beezhold DH. Lung epithelial cells resist influenza $A$ infection by inducing the expression of cytochrome c oxidase VIc which is modulated by miRNA 4276. Virology. 2014;468-470:256-264. doi:10.1016/j.virol.2014.08.007

63. Hamdan AM, Al-Gayyar MM, Shams MEE, et al. Thymoquinone therapy remediates elevated brain tissue inflammatory mediators induced by chronic administration of food preservatives. Sci Rep. 2019;9(1):7026. doi:10.1038/s41598-019-43568-x

64. Dahl O, Overgaard J. A Century With Hyperthermic Oncology In Scandinavia. Acta Oncologica. 1995;34(8):1075-1083. doi:10.3109/02841869509127234

65. Hildebrandt B. The cellular and molecular basis of hyperthermia. Crit Rev Oncol Hematol. 2002;43(1):33-56. doi:10.1016/S1040-8428(01)00179-2

66. De Haas-Kock DFM, Buijsen J, Pijls-Johannesma M, et al. Concomitant hyperthermia and radiation therapy for treating locally advanced rectal cancer. Cochrane Database Syst Rev.

2009;3:CD006269. doi:10.1002/14651858.CD006269.pub2

67. Lutgens $L$ et al. Combined use of hyperthermia and radiation therapy for treating locally advanced cervix carcinoma. Cochrane Database Syst Rev. 2010;3:CD006377.

68. Roizin-Towle L, Pirro JP. The response of human and rodent cells to hyperthermia. Int J Radiat Oncol Biol Phys. 1991;20(4):751-756. doi:10.1016/0360-3016(91)90018-Y

69. Tai W, He L, Zhang X, et al. Characterization of the receptor-binding domain (RBD) of 2019 novel coronavirus: implication for development of RBD protein as a viral attachment inhibitor and vaccine. Cell Mol Immunol. 2020;17(6):613-620. doi:10.1038/s41423-020-0400-4

70. Richter K, Haslbeck M, Buchner J. The heat shock response: life on the verge of death. Mol Cell. 2010;40(2):253-266. doi:10.1016/j.molcel.2010.10.006 
71. Nickavar B, Mojab F, Javidnia K, Amoli MAR. Chemical Composition of the Fixed and Volatile Oils of Nigella sativa L. from Iran. Z Naturforsch C. 2003;58(9-10):629-631. doi:10.1515/ znc-2003-9-1004

72. Baharetha HM, Nassar ZD, Aisha AF, et al. Proapoptotic and Antimetastatic Properties of Supercritical CO2 Extract of Nigella sativa Linn. Against Breast Cancer Cells.J Med Food. 2013;16(12):1121-1130. doi:10.1089/jmf.2012.2624

73. Ndreshkjana B, Çapci A, Klein V, et al. Combination of 5-fluorouracil and thymoquinone targets stem cell gene signature in colorectal cancer cells. Cell Death Dis. 2019;10(6):379.

doi:10.1038/s41419-019-1611-4

74. Fröhlich T, Reiter C, Saeed MEM, et al. Synthesis of Thymoquinone-Artemisinin Hybrids: New Potent Antileukemia, Antiviral, and Antimalarial Agents. ACS Med Chem Lett. 2018;9(6):534-539. doi:10.1021/acsmedchemlett.7b00412

75. Dajani E, Shahwan TG, Dajani NE. Overview of the Human Investigations of Nigella sativa (Black Seeds): A Complementary Drug with Historical and Clinical Significance. Gen Int Med Clin Innov. 2019;4(1). doi:10.15761/GIMCI.1000171

76. Beg S, Swain S, Rizwan Md, Irfanuddin Md, Shobha Malini D. Bioavailability Enhancement Strategies: Basics, Formulation Approaches and Regulatory Considerations. Curr Drug Deliv. 2011;8(6):691-702. doi:10.2174/156720111797635504

77. Tubesha Z, Imam M, Mahmud R, Ismail M. Study on the Potential Toxicity of a Thymoquinone-Rich Fraction Nanoemulsion in Sprague Dawley Rats. Molecules. 2013;18(7):7460-7472. doi:10.3390/molecules 18077460

78. Surekha $\mathrm{R}$ et al. An efficient encapsulation of thymoquinone using solid lipid nanoparticle for brain targeted drug delivery: physicochemical characterization, pharmacokinetics and biodistribution studies. Int J Pharm Clin Res. 8(12):1616-1624.

79. Goyal SN, Prajapati CP, Gore PR, et al. Therapeutic Potential and Pharmaceutical Development of Thymoquinone: A Multitargeted Molecule of Natural Origin. Front Pharmacol. 2017;8:656. doi:10.3389/fphar.2017.00656

80. Kalam MA, Raish M, Ahmed A, et al. Oral bioavailability enhancement and hepatoprotective effects of thymoquinone by self-nanoemulsifying drug delivery system. Mater Sci Eng C.

2017;76:319-329. doi:10.1016/j.msec.2017.03.088

81. Singh A, Ahmad I, Akhter S, et al. Nanocarrier based formulation of Thymoquinone improves oral delivery: Stability assessment, in vitro and in vivo studies. Colloids Surf B. 2013;102:822-832. doi:10.1016/j.colsurfb.2012.08.038

82. Elmowafy M, Samy A, Raslan MA, et al. Enhancement of Bioavailability and Pharmacodynamic Effects of Thymoquinone Via Nanostructured Lipid Carrier (NLC) Formulation. AAPS PharmSciTech. 2016;17(3):663-672. doi:10.1208/s12249-015-0391-0 83. Al-Ali A, Alkhawajah AA, Randhawa MA, Shaikh NA. Oral and intraperitoneal LD50 of thymoquinone, an active principle of Nigella sativa, in mice and rats.J Ayub Med Coll Abbottabad. 2008;20(2):25-27.

84. Abukhader M. The effect of route of administration in thymoquinone toxicity in male and female rats. Indian J Pharm Sci. 2012;74(3):195. doi:10.4103/0250-474X.106060

85. Abdelwahab SI, Sheikh BY, Taha MME, et al. Thymoquinone-loaded nanostructured lipid carriers: preparation, gastroprotection, in vitro toxicity, and pharmacokinetic properties after extravascular administration. Int J Nanomedicine. 2013;(8):2163-2172. 
86. Ong YS, Saiful Yazan L, Ng WK, et al. Acute and subacute toxicity profiles of thymoquinoneloaded nanostructured lipid carrier in BALB/c mice. Int J Nanomedicine. 2016;11:5905-5915. doi:10.2147/IJN.S114205

87. Sayed-Ahmed MM, Nagi MN. Thymoquinone supplementation prevents the development of gentamicin-induced acute renal toxicity in rats. Clin Exp Pharmacol Physiol. 2007;34(5-6):399-405. doi:10.1111/j.1440-1681.2007.04560.x

88. Badary OA, Nagi MN, Al-Shabanah OA, Al-Sawaf HA, Al-Sohaibani MO, Al-Bekairi AM. Thymoquinone ameliorates the nephrotoxicity induced by cisplatin in rodents and potentiates its antitumor activity. Can J Physiol Pharmacol. 1997;75(12):1356-1361. doi:10.1139/y97-169 